ISSN 1561-8358 (Print)

ISSN 2524-244X (Online)

УДК 536.46:534.29

https://doi.org/10.29235/1561-8358-2019-64-1-14-24

Поступила в редакцию 15.11.2018

Received 15.11.2018

\author{
В.В. Клубович ${ }^{1,3}$, М.М. Кулак ${ }^{2}$, Б.Б. Хина ${ }^{3}$ \\ ${ }^{1}$ Белорусский национальный технический университет, Минск, Беларусь \\ ${ }^{2}$ Институт технической акустики Национальной академии наук Беларуси, Витебск, Беларусь \\ ${ }^{3}$ Физико-технический институт Национальной академии наук Беларуси, Минск, Беларусь

\section{ОСОБЕННОСТИ ФОРМИРОВАНИЯ ОБЪЕМНЫХ ПОРИСТЫХ СИСТЕМ ПРИ САМОРАСПРОСТРАНЯЮЩЕМСЯ ВЫСОКОТЕМПЕРАТУРНОМ СИНТЕЗЕ. БОРИДЫ}

Аннотация. На разработанной экспериментальной установке исследовано влияние ультразвуковых колебаний (УЗК) на скорость и температуру горения, фазовый состав и микроструктуру соединений при самораспространяющемся высокотемпературном синтезе (CBC) в системе Тi-В. Показано, что влияние мощного ультразвука на СВС в данной системе связано как с тепловым фактором - повышением интенсивности теплоотвода от поверхности образца из-за вынужденной конвекции, так и с физическим (нетепловым) - воздействием УЗК на растекание расплава и кристаллизацию различных фаз в волне СВС.

Показано, что для многофазной системы титан-бор увеличение содержания бора в исходной шихте приводит к измельчению зерен в структуре синтезированного материала, а наложение ультразвуковых колебаний на процесс СВС приводит к изменению морфологии зерен: их форма становится более равноосной. В составе Тi+1,5B на внутренней поверхности пор наблюдается увеличение содержания зерен кубической формы, а в составе Тi $+2,0 \mathrm{~B}$ синтезированные зерна $\mathrm{TiB}_{2}$ приобретают более четкую огранку.

В результате наложения ультразвука на процесс синтеза для всех составов прослеживается измельчение зерен продукта наряду с образованием большого числа боридов и перераспределением их в объеме. Применение ультразвука приводит к изменению количественного фазового состава продуктов синтеза и соотношения между орторомбической и кубической модификациями фазы ТіВ. Проведенные исследования показали, что наличие пор в исходной шихте играет значительную роль в процессах структурообразования конечного продукта, и поэтому получить равновесный материал методом СВС невозможно. Установлено, что существует оптимальная амплитуда УЗК, при которой можно получить однородную мелкозернистую структуру материала, что позволяет управлять структурообразованием при СВС.

Ключевые слова: самораспространяющийся высокотемпературный синтез (CBC), ультразвуковые колебания (УЗК), скорость и максимальная температура горения, бориды титана, фазовый состав, микроструктура

Для цитирования: Клубович, В.В. Особенности формирования объемных пористых систем при самораспространяющемся высокотемпературном синтезе. Бориды / В.В.Клубович, М. М. Кулак, Б. Б. Хина // Вес. Нац. акад. навук Беларусі. Сер. фіз.-тэхн. навук. - 2019. - Т. 64, № 1. - С. 14-24. https://doi.org/10.29235/1561-8358-2019-64-1-14-24

\author{
V. V. Klubovich ${ }^{1,3}$, M. M. Kulak ${ }^{2}$, B. B. Khina ${ }^{3}$ \\ ${ }^{1}$ Belorussian National Technical University, Minsk, Belarus \\ ${ }^{2}$ Institute of Technical Acoustics of the National Academy of Sciences of Belarus, Vitebsk, Belarus \\ ${ }^{3}$ Physical-Technical Institute of the National Academy of Sciences of Belarus, Minsk, Belarus
}

\title{
SPECIFIC FEATURES OF FORMATION OF BULK POROUS SYSTEMS IN THE SELF-PROPAGATING HIGH- TEMPERATURE SYNTHESIS. BORIDES
}

\begin{abstract}
The effect of ultrasound oscillations (USO) on the combustion velocity and temperature, phase composition and microstructure of compounds produced by the self-propagating high-temperature synthesis (SHS) in the Ti-B system is studied using the earlier developed experimental setup. It is shown that the effect of powerful ultrasound on SHS is connected with the thermal factor, viz. the enhancement of heat removal from the specimen surface due to forced convection, and the physical (non-thermal) factor that consists in the influence of USO on the melt spreading and crystallization of different phases in the SHS wave. It is demonstrated that for multiphase system Ti-B an increase of the boron content in the initial charge leads to grain refinement of the synthesized product and the imposition of USO on the SHS results in a change in the grain morphology: their shape becomes closer to equiaxial. In composition $\mathrm{Ti}+1.5 \mathrm{~B}$, under the action of USO cubic-shape product grains are observed on the inner surface of pores, and in composition $\mathrm{Ti}+2.0 \mathrm{~B}$ the synthesized $\mathrm{TiB}_{2}$ grains become more vividly facetted.
\end{abstract}

(C) Клубович В.В., Кулак М. М., Хина Б. Б., 2019 
As a result of imposition of ultrasound on the synthesis process, grain refinement is observed for all the compositions along with the formation of a large number of borides and their redistribution in the volume. The use of ultrasound brings about changes in the quantitative phase composition of the synthesis products and the ratio between the orthorhombic and cubic modifications of phase TiB. The research has shown that the presence of pores in the initial charge plays an important role in the structure formation of the final product, and hence it appears impossible to obtain an equilibrium material by the SHS method. It is found that an optimal USO amplitude exists at which it is possible to obtain uniform fine-grained structure of the material. This permits controlling the structure formation at SHS.

Keywords: self-propagating high-temperature synthesis (SHS), ultrasound oscillations (USO), velocity and maximal temperature of combustion, titanium borides, phase composition, microstructure

For citation: Klubovich V. V., Kulak M. M., Khina B. B. Specific features of formation of bulk porous systems in the self-propagating high-temperature synthesis. Borides. Vestsi Natsyyanal'nai akademii navuk Belarusi. Seryya fizika-technichnych navuk = Proceedings of the National Academy of Sciences of Belarus. Physical-technical series, 2019, vol. 64, no. 1, pp. 14-24 (in Russian). https://doi.org/10.29235/1561-8358-2019-64-1-14-24

Введение. Отличительной чертой самораспространяющегося высокотемпературного синтеза (СBC) является то, что синтез конечных продуктов происходит по необычным (с точки зрения физического материаловедения), неравновесным механизмам (Явление волновой локализации автотормозящихся твердофазных реакций: диплом №287 СССР на открытие / А.Г. Мержанов, И. П. Боровинская, В.М. Шкиро; дата публ.: 30.08.1984, [1]). Этот факт выделяет процесс СВС в ряд перспективных технологий получения материалов в неравновесных условиях. Такие технологии открывают широкие возможности для создания различных материалов с заданной структурой и физико-механическими свойствами.

Макрокинетические характеристики процессов СВС, структура и свойства продуктов синтеза определяются множеством физических, технологических и химических свойств порошков исходных реагентов и их экзотермических смесей. Однако использование традиционных методик, предусматривающих регулирование процессов СВС путем изменения внешнего давления, начальной температуры, параметров смеси, нередко бывает ограничено. Согласно положениям синергетики [2], для неравновесной системы даже слабое воздействие может существенно изменить состояние такой системы. Поэтому для регулирования процессов горения и структурообразования конечных продуктов (даже in situ) необходимо использовать внешние физические воздействия. Одним из методов внешнего воздействия на процесс СВС является мощный ультразвук. В связи с этим следует ожидать, что наложение ультразвуковых колебаний (УЗК) на СВС-систему, в которой протекают неравновесные процессы химического взаимодействия и структурообразования, приведет к изменению ее фазового состава и структуры.

Материалы и методика эксперимента. В данной работе, которая является продолжением ранее выполненных исследований [3, 4], приведены полученные нами сведения о влиянии УЗК в СВС-системе Тi-В. Синтез проводили на разработанной установке (Установка для синтеза тугоплавких соединений: полезная модель 4319 Республика Беларусь: МПК (2006) В 22F 3/00 / В. В. Клубович, М. М. Кулак, Л. Л. Платонов; дата публ.: 30.04.2008) при различной амплитуде УЗК (६). Для приготовления исходной шихты использовали порошки титана с размером частиц $<50$ мкм и бора аморфного (удельная поверхность $16,5 \mathrm{~m}^{2} / \Gamma$ ). Образцы с составами $\mathrm{Ti}+\beta \mathrm{B}$, где $\beta$ - мольное соотношение компонентов, готовили по методике, описанной в [5]. В работе применяли металлографический, электронно-микроскопический, рентгеноструктурный и другие методы анализа фазового состава, структуры синтезированных материалов. Рентгеноструктурный (рентгенофазовый) анализ (РФА) проводили на дифрактометрическом комплексе D8 ADVANCE (BRUKER, Германия) в $\mathrm{CuK}_{\alpha}$ излучении. Массовое содержание фаз оценивали путем сравнения интенсивности их основных характеристических линий с эталоном. Исследование микроструктуры материалов проводили с использованием оптического микроскопа «МИКРО-200» и растрового сканирующего электронного микроскопа (СЭМ) марки LEO1455VP фирмы Carl Zeiss с последующей обработкой по программе количественной металлографии Image S.P. [6].

Результаты и их обсуждение. Такие характеристики волны CBC, как скорость и температура горения, зависят от начальной температуры шихты и условий теплообмена с окружающей средой. Поэтому тепловое влияние УЗК на СВС-процесс проявляется двояко: 1) разогрев шихты из-за поглощения энергии колебаний в порошковой среде и 2) более интенсивное охлаждение во 
время горения из-за вынужденной конвекции газовой среды вокруг осциллирующего образца. На рис. 1 приведены данные по оценке теплового воздействия УЗК на образцы состава Ті $+\beta B$ $(\beta=0,75 ; 1,0 ; 1,5 ; 2,0)$.
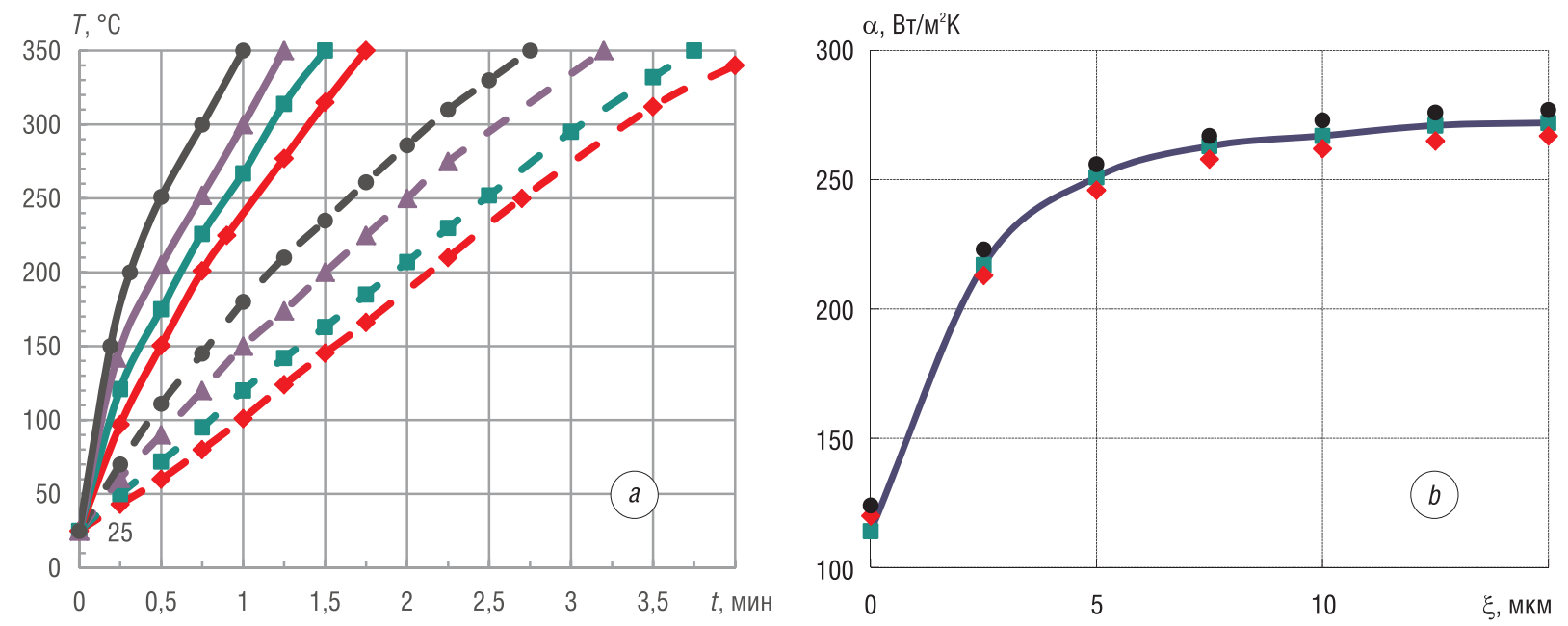

Рис. 1. Температура разогрева образцов смеси Ті $+\beta$ В с различным соотношением компонентов $\beta$ при разных амплитудах УЗК $\xi$ (пунктирные линии $-\xi=5$ мкм, сплошные $-\xi=10$ мкм) (a) и зависимость коэффициента теплоотдачи $\alpha$ на поверхности образцов с различным соотношением компонентов от амплитуды УЗК $\xi(b): \beta=>-0,75$, $-1,0, \mathbf{\Delta}-1,5, \boldsymbol{0}-2,0$

Fig. 1. The temperature of self-heating of specimens of the Ti+ $\beta \mathrm{B}$ mixtures with different component ratios $\beta$ for different USO amplitudes $\xi$ (dashed line $-\xi=5 \mu \mathrm{m}$; solid line $-\xi=10 \mu \mathrm{m})(a)$, and the heat transfer coefficient $\alpha$ on the surface of specimens with different component ratios vs. the USO amplitude $\xi(b): \beta=-0.75, \square-1.0, \boldsymbol{\Delta}-1.5, \bullet-2.0$

Из приведенных на рис. 1, а данных видно, что температура разогрева и скорость ее нарастания существенно зависят как от амплитуды подводимых колебаний, так и от состава исходной смеси, и они значительно меньше скоростей и температур разогрева, достигаемых при процессе СВС в обычных условиях без УЗК. Из сравнения времени разогрева со временем протекания процесса синтеза можно сделать вывод, что при проведении синтеза с наложением УЗК процесс протекает при постоянной начальной температуре.

Были рассчитаны коэффициенты теплоотдачи для исследуемых систем. Результаты приведены на рис. $1, b$. Видно, что наложение УЗК приводит к увеличению коэффициента теплоотдачи, а величина коэффициента теплоотдачи не зависит от состава исходной шихты. Это связано, по-видимому, с ростом интенсивности конвективных потоков под действием УЗК вблизи поверхности горящего образца, что может влиять на образование конечного продукта.

Зависимости температуры и скорости горения от амплитуды УЗК для составов Тi $+\beta B$ приведены на рис. 2.

Наблюдаемое уменьшение скорости и температуры горения при наложении УЗК на СВСпроцесс в данной системе согласуется с положениями классической теории горения [7]. Это связано, вероятнее всего, с тепловым фактором - охлаждением образца из-за вынужденной конвекции окружающего инертного газа (аргона) в результате колебаний образца. Однако может также иметь место физическое (нетепловое) влияние ультразвука, которое должно проявляться в изменении состава продукта взаимодействия в волне СВС и его микроструктуры. Для анализа роли этого фактора нами были выполнены рентгеновские и микроструктурные исследования.

При проведении рентгенофазового анализа образцов, синтезированных при воздействии УЗК, было отмечено изменение ширины и интегральной интенсивности дифракционных линий в зависимости от амплитуды приложенных колебаний и состава исходной шихты. Поэтому нами был исследован фазовый состав продуктов синтеза с использованием РФА, а также проведен анализ микроструктуры. Массовые доли фаз определяли пропорционально интенсивности их характеристических линий. Результаты РФА приведены в таблице. 
$T, \mathrm{~K}$

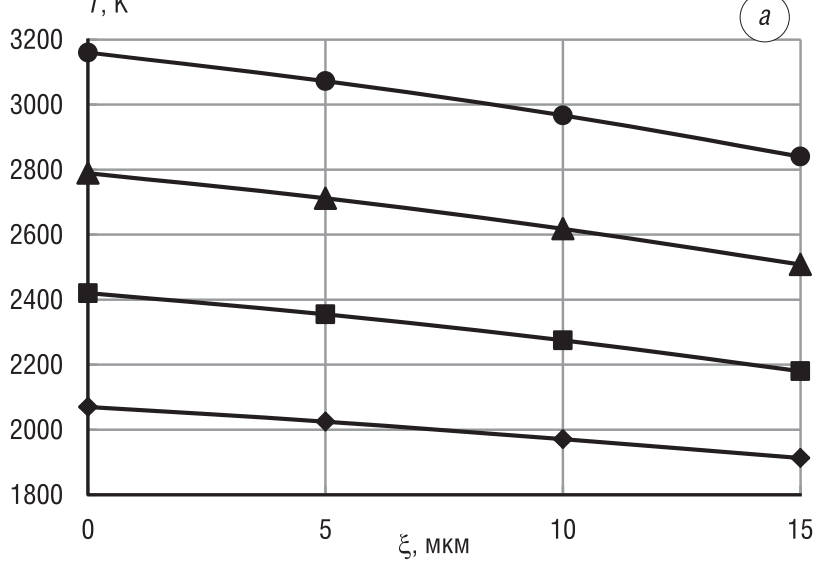

$U, \mathrm{~cm} / \mathrm{c}$

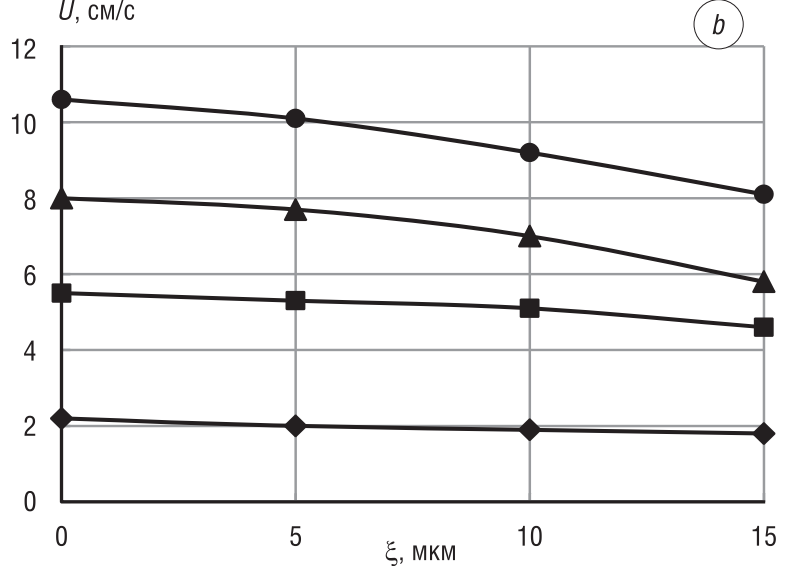

Рис. 2. Зависимости температуры $(a)$ и скорости $(b)$ горения составов системы титан-бор с различным мольным соотношением компонентов от амплитуды УЗК: $\diamond-\beta=0,75, \boldsymbol{\square}-\beta=1,0, \boldsymbol{\Delta}-\beta=1,5, \bullet-\beta=2,0$

Fig. 2. Combustion temperature $(a)$ and velocity $(b)$ of the titanium-boron compositions with different molar ratio of components vs. the USO amplitude: $\diamond-\beta=0.75, \boldsymbol{\square}-\beta=1.0, \boldsymbol{\Delta}-\beta=1.5, \boldsymbol{\bullet}-\beta=2.0$

Полуколичественный фазовый анализ продуктов горения составов Тi+ $\beta$ B Semi-quantitative phase analysis of combustion products in the $\mathrm{Ti}+\beta \mathrm{B}$ compositions

\begin{tabular}{|c|c|c|c|c|c|c|}
\hline$\beta$ & $\xi$, мкм & $\begin{array}{c}\text { TiB } \\
\text { (орторомбическая модификация), \% }\end{array}$ & $\begin{array}{c}\text { TiB } \\
\text { (кубическая модификация), \% }\end{array}$ & $\mathrm{TiB}_{2}, \%$ & $\mathrm{Ti}, \%$ & $\mathrm{Ti}_{3} \mathrm{~B}_{4}, \%$ \\
\hline \multirow[t]{3}{*}{0,75} & 0 & 73,4 & 4,6 & 3,1 & 18,9 & 0 \\
\hline & 5 & 69,2 & 9,4 & 3,5 & 17,9 & 0 \\
\hline & 10 & 68,3 & 9,1 & 6,6 & 16 & 0 \\
\hline \multirow[t]{3}{*}{1,0} & 0 & 78,2 & 9,2 & 5,4 & 0,9 & 6,3 \\
\hline & 5 & 80,3 & 8,3 & 5,8 & 0,5 & 5,1 \\
\hline & 10 & 82,9 & 6,8 & 6,1 & 0,2 & 4 \\
\hline \multirow[t]{3}{*}{1,5} & 0 & 22,8 & 7,6 & 58,8 & 0,8 & 10 \\
\hline & 5 & 20,6 & 7,2 & 61,9 & 0,6 & 9,7 \\
\hline & 10 & 19,8 & 5,3 & 66,9 & 0,5 & 7,5 \\
\hline \multirow[t]{3}{*}{2,0} & 0 & - & - & 100,0 & - & - \\
\hline & 5 & - & - & 100,0 & - & - \\
\hline & 10 & - & - & 100,0 & - & - \\
\hline
\end{tabular}

Во всех исследованных ситуациях температура СВС превышает точку плавления титана $\left(T_{m}(\mathrm{Ti})=1941 \mathrm{~K}\right)($ рис. $2, a)$, но она ниже температуры плавления кристаллического бора $\left(T_{m}(\mathrm{~B})=\right.$ $2365 \mathrm{~K}$ ) для состава Ті+0,75В при всех амплитудах УЗК ( $\xi=0-15$ мкм). Температура горения становится ниже точки плавления бора для состава с $\beta=1,5$ при $\xi>10$ мкм и для состава с $\beta=1,0$ при $\xi>5$ мкм.

При $T_{\mathrm{CBC}}>T_{m}(\mathrm{~B})($ состав с $\beta=1,0$ без УЗК и состав с $\beta=1,5$ при $\xi \leqslant 10$ мкм) продукт кристаллизуется из уже имеющегося бинарного расплава титан-бор. Однако в ситуациях, когда $T_{\mathrm{CBC}}<$ $T_{m}(\mathrm{~B})$ (составы с $\beta=0,75$ при всех $\xi, \beta=1,5$ при $\xi>10$ мкм и $\beta=1,0$ при $\xi>5$ мкм) в высокотемпературной зоне волны СВС происходят растворение твердого бора в жидком титане и кристаллизация зерен продукта из расплава. Поэтому в ситуациях, когда $T_{\mathrm{CBC}}<T_{m}(\mathrm{~B})$, влияние ультразвука на СВС-процесс может проявляться в изменении кинетики растворения бора в жидком титане и условий кристаллизации зерен продукта из расплава.

Для состава с $\beta=2,0$ единственным продуктом является фаза $\mathrm{TiB}_{2}$, и $T_{\mathrm{CBC}}>T_{m}(\mathrm{~B})$ при всех амплитудах УЗК. Термодинамический расчет с использованием данных [8] показал, что адиабатическая температура СВC $T_{a d}$ для данного состава совпадает с точкой плавления указанной фазы: $T_{a d}=T_{m}\left(\mathrm{TiB}_{2}\right)=3476 \mathrm{~K}$, при этом доля расплава составляет 10,6 \%, то есть энтальпии экзотермической реакции образования $\mathrm{TiB}_{2}$ недостаточно для полного расплавления продукта. 
В составе с $\beta=1,5$ при всех амплитудах УЗК $T_{\mathrm{CBC}}>T_{m}$ (B) (рис. $2, a$ ), и поэтому зерна самой тугоплавкой фазы $\mathrm{TiB}_{2}\left(T_{m}\left(\mathrm{TiB}_{2}\right)>T_{\mathrm{CBC}}\right)$ кристаллизуются из бинарного расплава $\mathrm{Ti}-\mathrm{B}$ непосредственно в зоне высокотемпературной реакции волны СВС. Однако основным равновесным продуктом для этого состава является фаза $\mathrm{Ti}_{3} \mathrm{~B}_{4}$, для которой $T_{m}\left(\mathrm{Ti}_{3} \mathrm{~B}_{4}\right)=2473 \mathrm{~K}>T_{\mathrm{CBC}}$ при $\xi \leqslant 5$ мкм. Поэтому эта фаза будет кристаллизоваться из расплава при остывании в зоне догорания волны $\mathrm{CBC}$ при $\xi \leqslant 5$ мкм и непосредственно в зоне высокотемпературной реакции при $\xi>5$ мкм.

Для состава с $\beta=1,0$ основным продуктом ( $\approx 90 \%)$ является фаза $\mathrm{TiB}$, для которой $T_{m}(\mathrm{TiB}=$ $2453 \mathrm{~K}$ ), и присутствует около $5 \% \mathrm{Ti}_{3} \mathrm{~B}_{4}$. При этом температура СВС при всех значениях амплитуды колебаний ниже точек плавления указанных фаз, то есть они кристаллизуются из расплава в зоне реакции волны СВС. Отметим, что без УЗК, когда $T_{\mathrm{CBC}}>T_{m}(\mathrm{~B})$ (см. рис. $\left.2, a\right)$, продукт кристаллизуется из бинарного расплава Тi-B, то есть растворение бора в жидком титане не может лимитировать процесс. Но при наложении УЗК ( $\xi>5$ мкм) температура СВС становится ниже точки плавления бора. Тогда зерна $\mathrm{TiB}_{\text {и }} \mathrm{Ti}_{3} \mathrm{~B}_{4}$ будут кристаллизоваться из расплава по мере растворения твердого бора в жидком титане.

Для состава с $\beta=0,75$ основным продуктом является фаза ТіВ и остается избыток титана; при этом $T_{\mathrm{CBC}}<T_{m}(\mathrm{~B})$ при всех условиях проведения СВС (с УЗК и без него). Следовательно, фаза ТiВ может кристаллизоваться из расплава в зоне реакции волны СВС только по мере растворения твердого бора в жидком титане.

С помощью программы Image S.P. [6] по методике, описанной в [3], проведен количественный анализ соотношения металлокерамической компоненты (МКК) D и пор в продольном осевом сечении синтезированного материала по величине занимаемой ими площади. Сначала обсчитывали площадь долевого сечения образца $\left(S_{\text {комп. }}\right)$, затем - площадь того же сечения без учета пор $\left(S_{\text {мет. }}\right)$. Относительная доля площади металлизированной компоненты $\mathbf{D}$ в сечении составляла $\left(S_{\text {мет. }} /\left(S_{\text {комп. }}\right) \times 100 \%\right.$. Результаты приведены на рис. $3, a$. Видно, что наложение УЗК на $\mathrm{CBC}$-процесс приводит к увеличению относительной плотности (то есть снижению пористости) и более однородному распределению пор по высоте. Таким образом, УЗК позволяет повысить однородность микроструктуры полученного композиционного материала состава $\mathrm{Ti}+1,0 \mathrm{~B}$, в который входят $\mathrm{TiB}, \mathrm{TiB}_{2}, \mathrm{Ti}_{3} \mathrm{~B}_{4}$ и остаточный титан (см. таблицу).

На рис. $3, b$ приведена зависимость $\mathbf{D}$ в центральном поперечном сечении от амплитуды УЗК для синтезированных материалов с составами Ti+1,0B, Ti+1,5B и Ti+2,0B. Видно, что с увеличением амплитуды УЗК до 10 мкм наибольшее уменьшение пористости имеет место для состава $\mathrm{Ti}+1,5 \mathrm{~B}$ - почти на $8 \%$; для состава Тi $+1,0 \mathrm{~B}$ пористость снижается на $5 \%$, а в составе Тi $+2,0 \mathrm{~B}$ изменение менее выражено. По-видимому, это различие связано с разным фазовым составом СВСпродукта для указанных образцов.
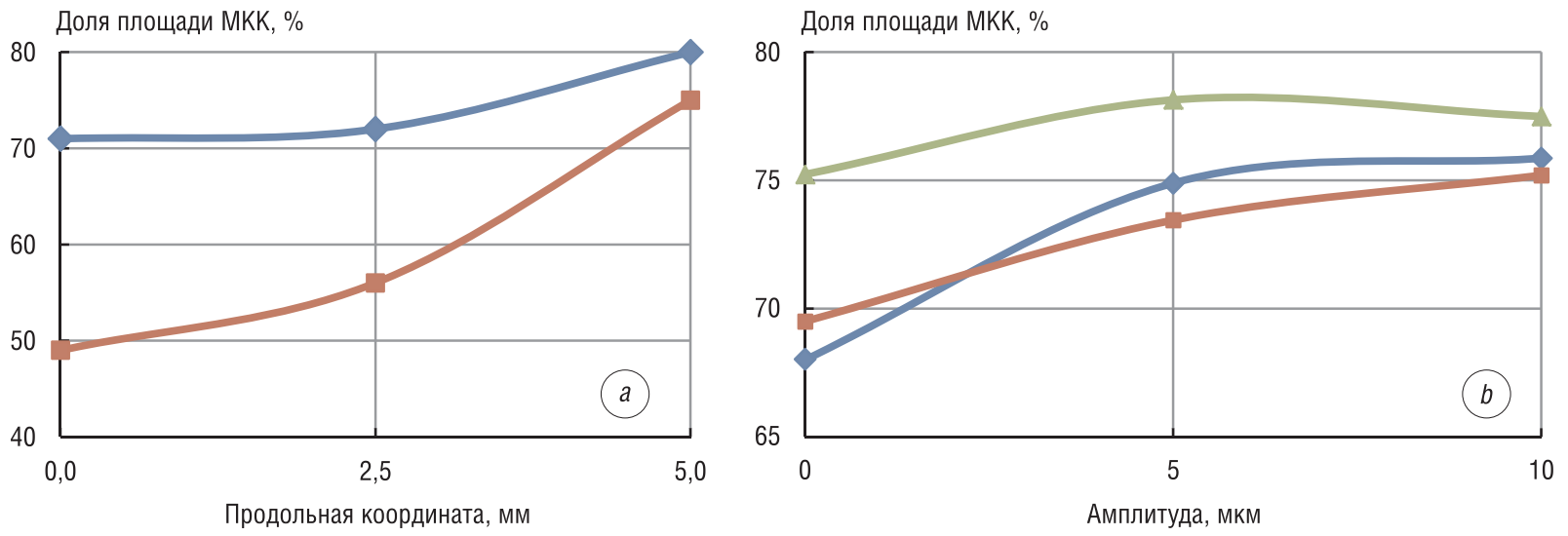

Рис. 3. Изменение относительной площади, занимаемой МКК в синтезированном материале: $a-$ состава Тi+1,0B по

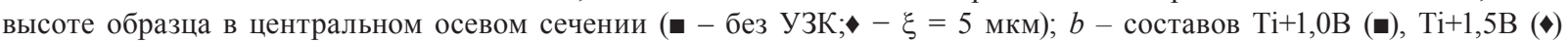
и $\mathrm{Ti}+2,0 \mathrm{~B}(\boldsymbol{\Delta})$ в центральном поперечном сечении

Fig. 3. Variation of the relative surface area occupied by the metal-ceramic phase: $a$-over the specimen height in the central

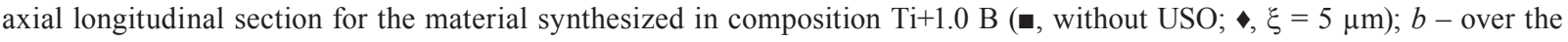
central transverse section for the materials synthesized in compositions $\mathrm{Ti}+1.0 \mathrm{~B}(\mathbf{\bullet}), \mathrm{Ti}+1.5 \mathrm{~B}(\bullet)$ and $\mathrm{Ti}+2.0 \mathrm{~B}(\mathbf{\Delta})$ 
На рис. 4 показаны результаты количественного металлографического анализа пористой структуры материалов составов $\mathrm{Ti}+1,0$ В и Тi $+2,0 \mathrm{~B}$ - распределения пор по среднему размеру при СВС без ультразвука и с УЗК амплитудой $\xi=5$ мкм.

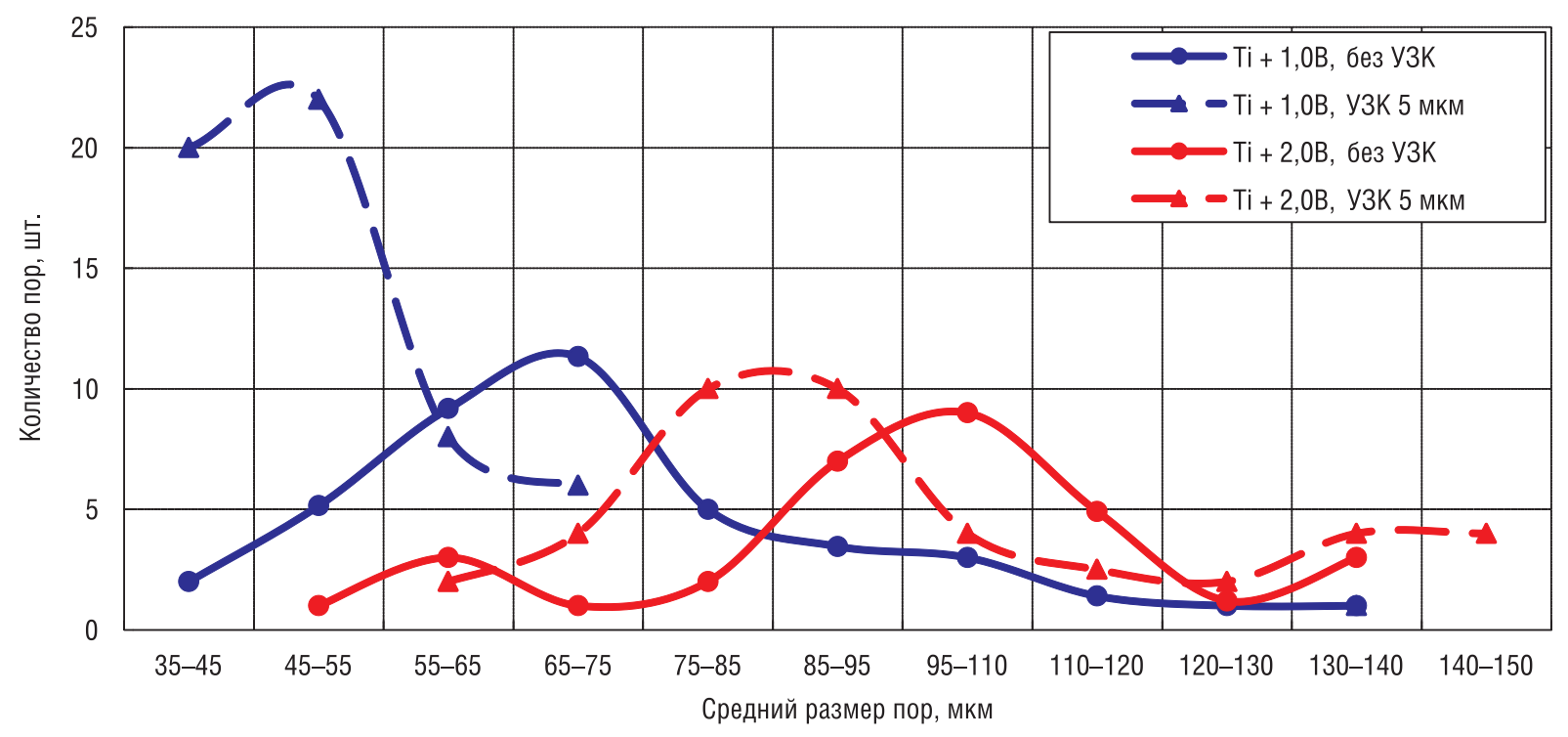

Рис. 4. Распределение пор по среднему размеру в синтезированных материалах составов Тi $+1,0 \mathrm{~B}$ и $\mathrm{Ti}+2,0 \mathrm{~B}$ после СВС без и с УЗК амплитудой $\xi=5$ мкм

Fig. 4. Distribution of pores by average size in the materials produced by SHS in compositions $\mathrm{Ti}+1.0 \mathrm{~B}$ and $\mathrm{Ti}+2.0 \mathrm{~B}$ without USO and with USO at amplitude $\xi=5 \mu \mathrm{m}$

Наложение УЗК приводит к смещению максимумов на кривых в сторону меньших размеров. При этом для состава Тi $+1,0 \mathrm{~B}$ наблюдается не только снижение среднего диаметра пор, но и значительное уменьшение их разброса по размеру при $\xi=5$ мкм - на кривой возникает острый максимум при размере пор около 45 мкм. Следовательно, наложение УЗК на СВС-процесс в данных составах позволяет управлять относительной плотностью синтезированного материала, распределением и средним размером пор. Эти параметры определяют ряд важных функциональных свойств материала.

Проведение количественного металлографического анализа структуры CBC-продуктов в данной системе осложняется тем, что из-за высокой химической стабильности боридов титана на микрошлифах поперечного среза образцов не удается выявить границы зерен с помощью травителей, используемых в металлографии. Поэтому анализ поперечных изломов (сколов) синтезированных образцов выполнялся на СЭМ с последующей обработкой изображений по программе Image S.P. Зеренная структура сколов не была выявлена, она видна только вблизи пор при большем увеличении.

Микроструктура (СЭМ) образца состава Тi+1,0B, полученного путем СВС без УЗК, ее обработка с помощью программы Image S.P. и построенная гистограмма распределения размеров зерен продукта показаны на рис. 5. Видно, что зерна боридов в выбранной области имеют округлую форму и почти одинаковый размер - 3-4 мкм (рис. 5, a). При наложении УЗК в процессе синтеза ( $\xi=5$ и 10 мкм) зеренная структура CВС-продукта не выявляется на СЭМ-изображениях даже при увеличении в 5000 раз (рис. 5). Это может быть связано с растеканием непрореагировавшего титана, содержание которого в продукте составляет около 20 \% (см. таблицу).

С помощью СЭМ были исследованы образцы различного стехиометрического состава, полученные при СВС без наложения и с наложением ультразвука. Съемка проводилась на поперечном сколе. Различие морфологических форм боридов титана обусловлено разнообразием образующихся соединений.

Бориды исходного состава Ti+1,0B и Тi+2,0B имеют округлую форму, бориды для состава $\mathrm{Ti}+1,5 \mathrm{~B}$ - кубическую. В результате наложения ультразвуковых колебаний для состава $\mathrm{Ti}+1,5 \mathrm{~B}$ на внутренней поверхности пор наблюдается увеличение содержания зерен продукта кубической 

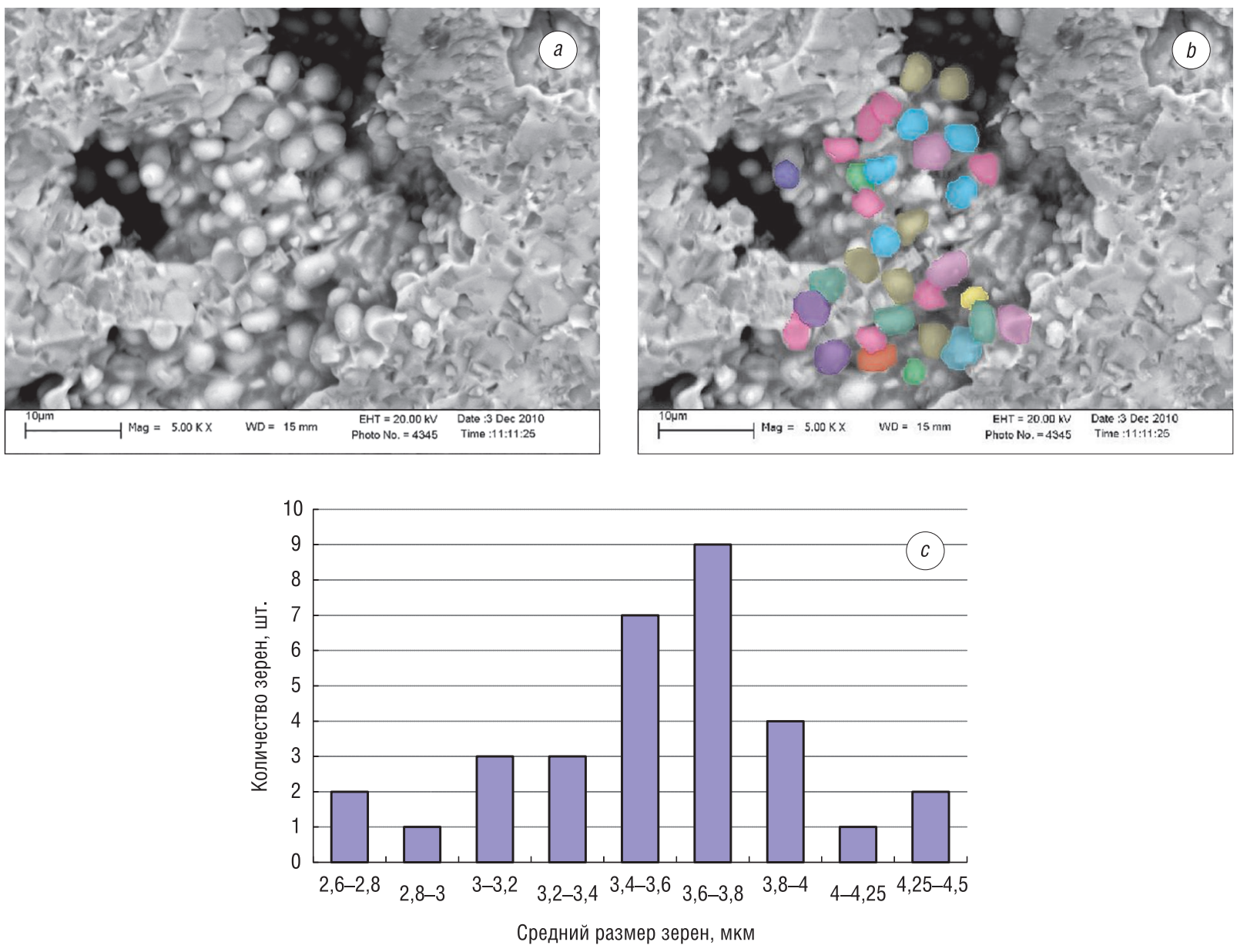

Рис. 5. Количественная металлография образца состава Тi+1,0В (СВС без УЗК): $a$ - СЭМ, $b$ - наложение масок, $c-$ гистограмма распределения зерен по размеру

Fig. 5. Quantitative metallography of the specimen synthesized in composition Ti+1.0B (SHS without USO): $a-\mathrm{SEM}, b-$ masking, $c$ - histogram of the grain size distribution

формы (рис. 6), а структура зерен состава Ті+2,0B приобретает более четкую огранку (рис. 7). Проведенным рентгеноструктурным анализом подтверждено наличие в данных образцах следующих фаз: $\mathrm{Ti}, \mathrm{TiB}$ - орторомбическая и кубическая, $\mathrm{Ti}_{3} \mathrm{~B}_{4}$ и $\mathrm{TiB}_{2}$ - гексагональная.

В результате наложения ультразвука на процесс синтеза для всех составов прослеживается измельчение фазовых составляющих с образованием большого числа новых боридов и перераспределением их в объеме. Наложение ультразвука приводит к изменению как фазового, так и количественного состава синтезированных фаз.
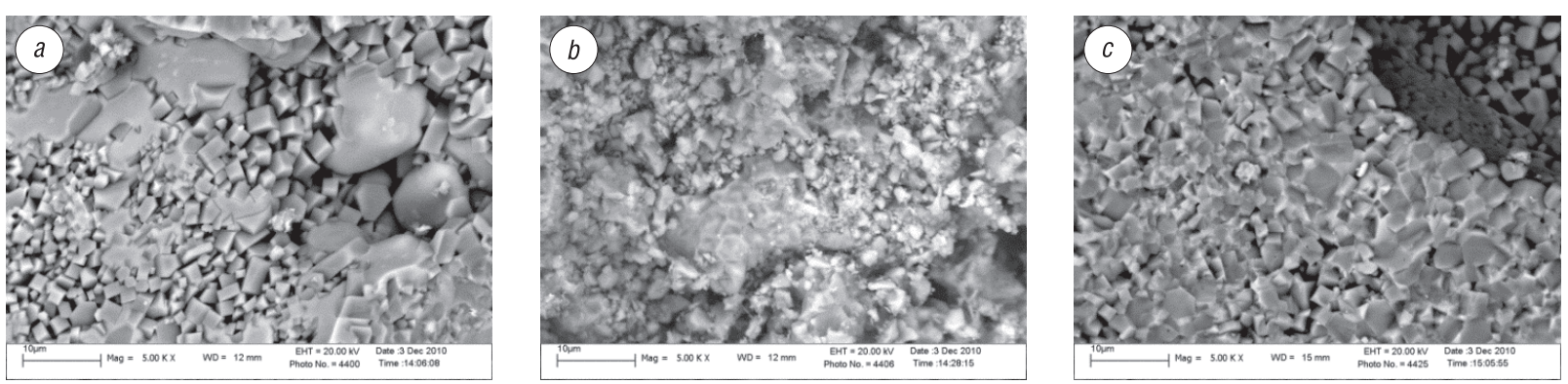

Рис. 6. Микроструктура материала состава Ті $+1,5 \mathrm{~B}$ после СВС: $a-\xi=0 ; b-\xi=5$ мкм; $c-\xi=10$ мкм

Fig. 6. Microstructure of the material synthesized in composition Ti+1.5B by SHS: $a-\xi=0 ; b-\xi=5 \mu \mathrm{m} ; c-\xi=10 \mu \mathrm{m}$ 

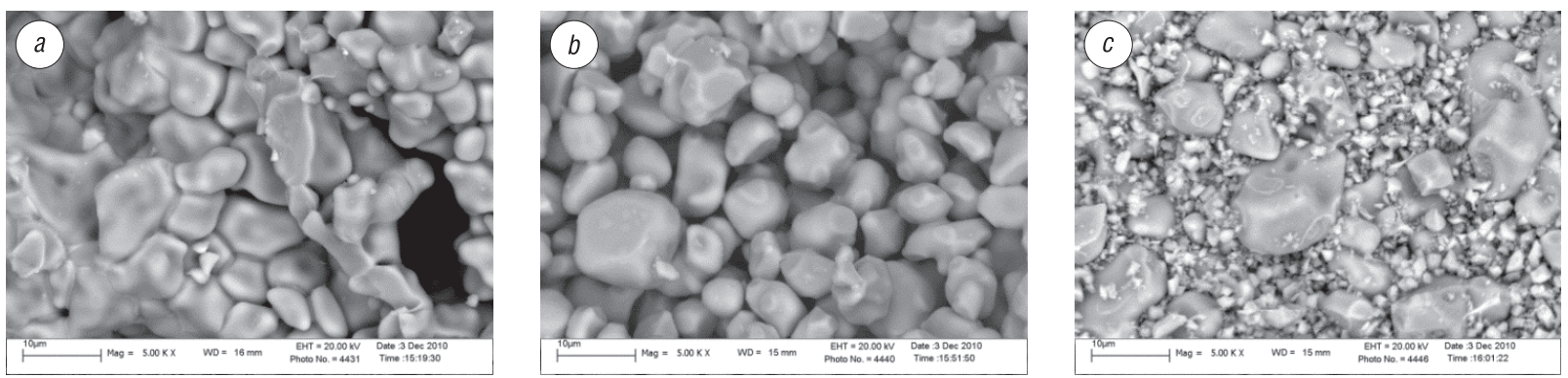

Рис. 7. Микроструктура материала состава Ті $+2,0 \mathrm{~B}$ после СВС: $a-\xi=0 ; b-\xi=5$ мкм; $c-\xi=10$ мкм

Fig. 7. Microstructure of the material synthesized in composition Ti+2.0B by SHS: $a-\xi=0 ; b-\xi=5 \mu \mathrm{m} ; c-\xi=10 \mu \mathrm{m}$

Проведенные исследования показали, что наличие пор в исходной шихте играет значительную роль в процессах структурообразования конечного продукта, и поэтому получить равновесный материал методом СВС невозможно.

На гистограмме (рис. 8) приведены средние размеры зерен СВС-продукта для шихты состава $\mathrm{Ti}+1,5 \mathrm{~B}$.

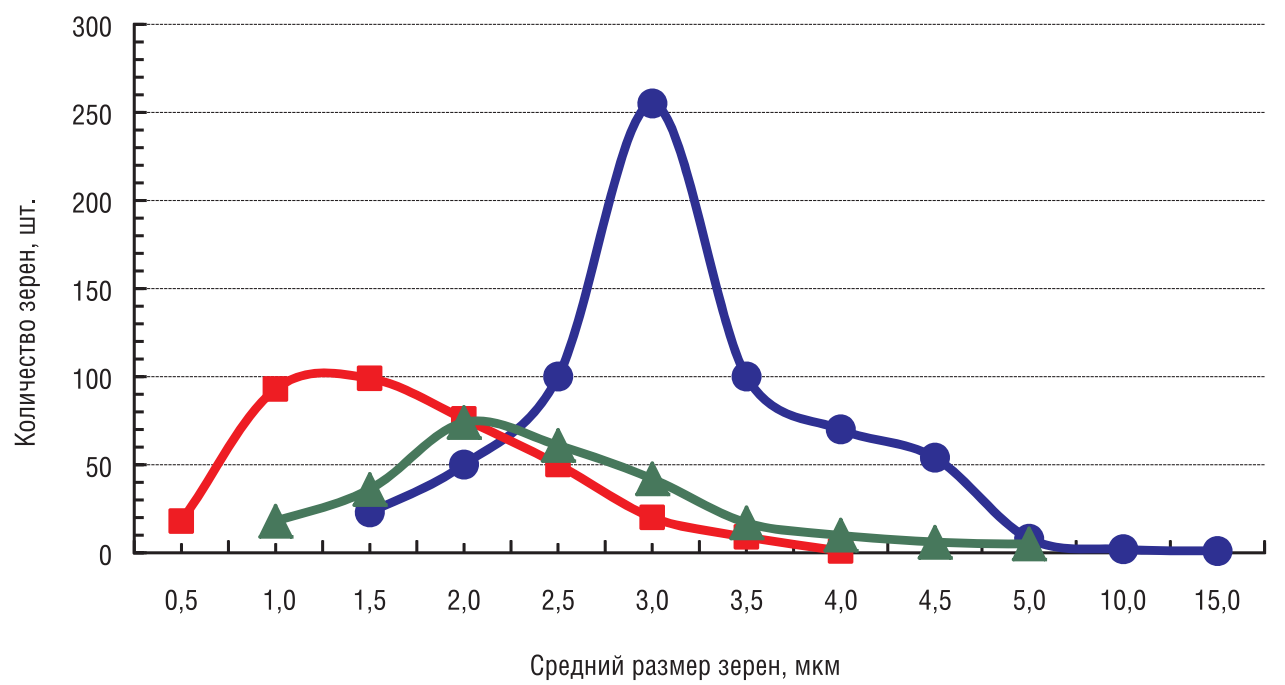

Рис. 8. Суммарная гистограмма среднего размера зерен СВС-продукта в шихте состава Тi+1,5В при разных амплитудах УЗК $\xi: \bullet-\xi=0 ; \boldsymbol{-}-\xi=5$ мкм; $\boldsymbol{\Delta}-\xi=10$ мкм

Fig. 8. Summary histogram of the average grain size distribution in the SHS product for charge composition Ti+1.5B at different USO amplitudes: $\bullet-\xi=0 ; \boldsymbol{\square}-\xi=5 \mu \mathrm{m} ; \boldsymbol{\Delta}-\xi=10 \mu \mathrm{m}$

Видно, что наложение УЗК на СВС-процесс в шихте указанного состава приводит к изменению распределения зерен продукта по среднему размеру. При этом существует некоторая амплитуда (здесь $\xi=5$ мкм), когда максимум на гистограмме смещается в сторону меньших диаметров зерен. Это открывает возможность управления структурой СВС-продукта, то есть «подстройкой» ее под конкретное функциональное назначение синтезируемого материала.

Аналогичное исследование проведено для микроструктур, полученных на СЭМ для состава шихты Ті $+2,0 \mathrm{~B}$ после синтеза с разными амплитудами УЗК. На рис. 9 приведены гистограммы среднего размера зерен СВС-продукта в шихте состава Ті+2,0В при разных амплитудах УЗК. Продукт на $100 \%$ состоит из зерен $\mathrm{TiB}_{2}$ (см. таблицу).

В отсутствие УЗК средний размер большинства зерен лежит в интервале 4,5-8,5 мкм (рис. 9, a). При наложении на СBC-процесс ультразвука с амплитудой 5 мкм интервал размеров, в который попадает большинство зерен, составляет 3,0-8,0 мкм (рис. 9, b), то есть практически не меняется. Однако при увеличении амплитуды УЗК до 10 мкм микроструктура претерпевает изменение: в ней присутствует небольшое количество зерен со средним диаметром 5,0 8,0 мкм, но подавляющее большинство зерен имеет малый размер - от 0,5 до 2,0 мкм (рис. 9, c). 

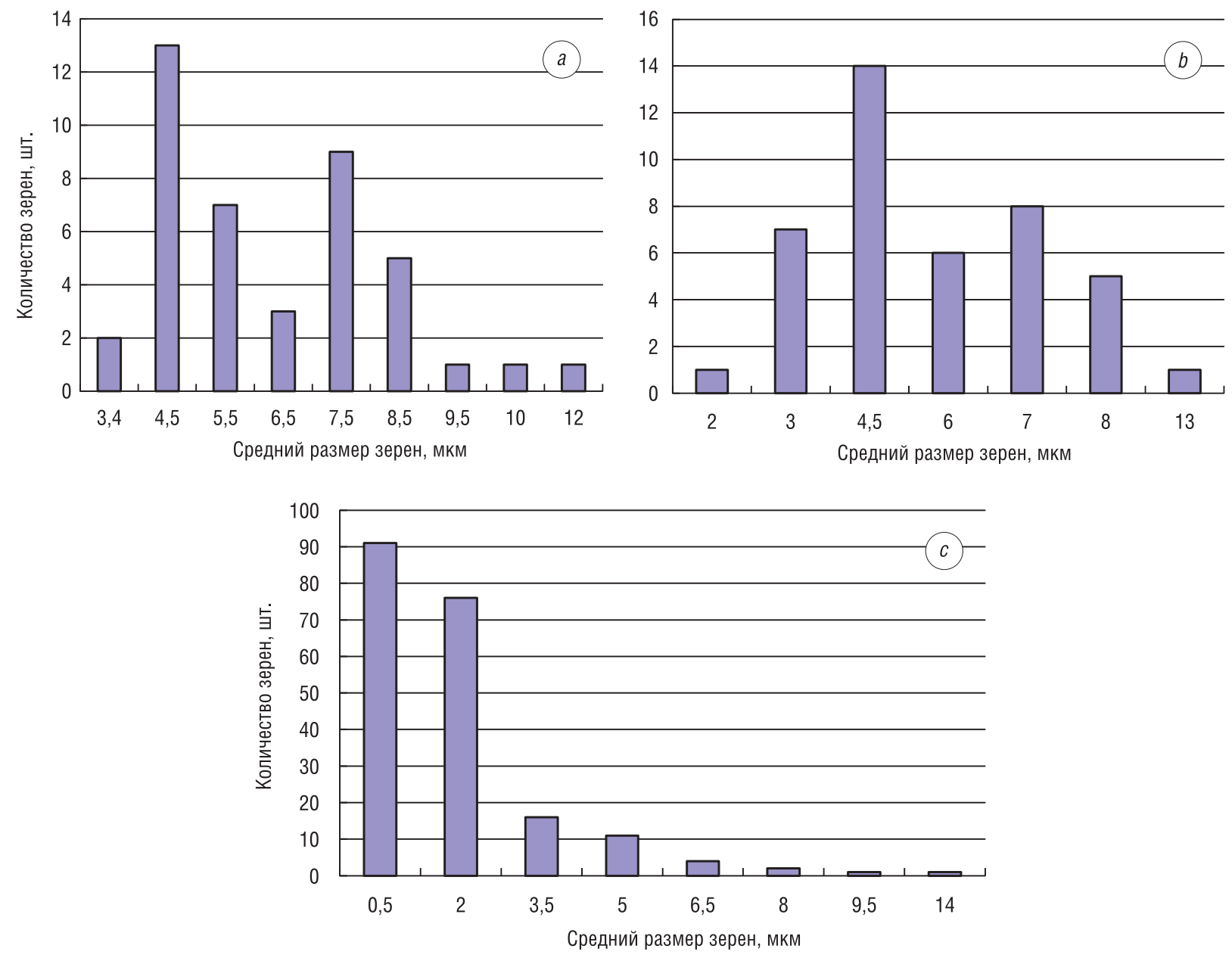

Рис. 9. Гистограмма среднего размера зерен на сколе материала состава Тi $+2,0 \mathrm{~B}$ после СВС: $a-\xi=0 ; b-\xi=5$ мкм; $c-\xi=10$ мкм

Fig. 9. Histogram of the average grain size over the fracture surface for charge composition Ti+2.0B after SHS: $a-\xi=0$; $b-\xi=5 \mu \mathrm{m} ; c-\xi=10 \mu \mathrm{m}$

Следовательно, для данного состава шихты амплитуда УЗК, обеспечивающая наибольшее изменение микроструктуры CBC-продукта (твердой тугоплавкой фазы $\mathrm{TiB}_{2}$ ), составляет $\xi=10$ мкм. Поскольку температура СВС для данного состава высока и единственным продуктом взаимодействия является соединение $\mathrm{TiB}_{2}$, влияние УЗК на $\mathrm{CBC}$ связано только с физическим воздействием на растекание расплава в волне СВС и его кристаллизацию.

Заключение. Влияние мощного ультразвука на СBC в системе Тi-В связано как с тепловым фактором - повышением интенсивности теплоотвода от поверхности образца из-за вынужденной конвекции, так и с физическим (нетепловым) фактором - воздействием УЗК на растекание расплава и кристаллизацию различных фаз в волне СВС.

Показано, что для многофазной системы титан-бор увеличение содержания бора в исходной шихте приводит к измельчению зерен в структуре синтезированного материала, а наложение ультразвуковых колебаний на процесс $\mathrm{CBC}-$ к изменению морфологии зерен: их форма становится более равноосной, размер уменьшается с 12-14 мкм до 8-10 мкм и разброс по размеру снижается. Наложение УЗК на процесс горения смесей с многофазным конечным продуктом при мольном соотношении реагентов 0,$75 ; 1,0 ; 1,5 ; 2,0$ и 2,25 приводит к изменению фазового состава продуктов синтеза. Для всех исследованных составов наложение УЗК приводит к увеличению содержания фазы $\mathrm{TiB}_{2}$. Для состава $\mathrm{Ti}+0,75 \mathrm{~B}$ обнаружены: фаза титана, ТiB с орторомбической и кубической решетками и фаза $\mathrm{TiB}_{2}$. При горении составов $\mathrm{Ti}+1,0 \mathrm{~B}$ и $\mathrm{Ti}+1,5 \mathrm{~B}$ кроме перечисленных фаз обнаружена фаза $\mathrm{Ti}_{3} \mathrm{~B}_{4}$, а при горении составов $\mathrm{Ti}+2,0 \mathrm{~B}$ и $\mathrm{Ti}+2,25 \mathrm{~B}$ образуется только фаза $\mathrm{TiB}_{2}$. В результате наложения ультразвуковых колебаний в боридной системе исходного 
состава $\mathrm{Ti}+1,5 \mathrm{~B}$ на внутренней поверхности пор наблюдается увеличение содержания продукта кубической формы TiB, а структура зерен боридной системы состава Ti+2,0B приобретает более четкую огранку.

Установлено, что существует оптимальная амплитуда УЗК (для состава Ti+1,5 В это 5 мкм, а для $\mathrm{Ti}+2,0 \mathrm{~B}-\xi=10$ мкм), при которой можно получить однородную мелкозернистую структуру материала. Это позволяет управлять структурообразованием при СВС.

Таким образом, в результате проведенных исследований можно сделать вывод, что наложение УЗК на СВС процесс позволяет in situ изменять фазовый состав и микроструктуру продукта (относительную плотность, размер, морфологию и распределение пор, размер зерен фаз и их распределение по среднему диаметру) в системе Ті-В. Это открывает перспективу модифицирования СВС-технологий получения новых пористых материалов на основе боридов титана с целью «подстройки» микроструктуры получаемого продукта под конкретную функцию материала. То есть наложение УЗК на СВС-процесс является эффективным физическим методом целенаправленного регулирования состава и структуры конечных продуктов синтеза и может быть использовано в качестве средства управления процессом синтеза.

\section{Список использованных источников}

1. К вопросу о равновесности продуктов СВС / А. Г. Мержанов [и др.] // Докл. Акад. наук. - 2004. - Т. 394, №4. C. $498-502$.

2. Николис, Г. Самоорганизация в неравновесных системах: от диссипативных структур к упорядоченности через флуктуации / Г. Николис, И. Пригожин. - М.: Мир, 1979. - 512 с.

3. Клубович, В.В. Особенности формирования объемных пористых систем при СВС-процессе. Силициды / В. В. Клубович, М. М. Кулак, И. Н. Румянцева // Вес. Нац. акад. навук Беларусі. Сер. фіз.-тэхн. навук. - 2010. - № 4. C. $5-10$.

4. Клубович, В.В. Особенности формирования объемных пористых систем при СВС-процессе. Карбиды / В. В. Клубович, М.М. Кулак, И.Н. Румянцева // Вес. Нац. акад. навук Беларусі. Сер. фіз.-тэхн. навук. - 2011. - № 1. C. $5-10$.

5. Клубович, В.В. Ультразвук в процессах СВС / В.В.Клубович, М.М. Кулак, Б.Б. Хина. - Минск: БНТУ, 2006. $-279 \mathrm{c}$.

6. Image S.P. SYSPROG Software Development Company [Электронный ресурс]. - Режим доступа: http://www.sysprog.com - Дата доступа: 16.08.2018.

7. Математическая теория горения и взрыва / Я. Б. Зельдович [и др.]. - М.: Наука, 1980. - 478 с.

8. The Al-B-Nb-Ti system: I. Re-assessment of the constituent binary systems B-Nb and B-Ti on the basis of new experimental data / V. T. Witusiewicz [et al.] // Journal of Alloys and Compounds. - 2008. - Vol. 448, iss. 1-2. - P. $185-194$. https://doi.org/10.1016/j.jallcom.2006.10.034

\section{References}

1. Merzhanov A. G., Kovalev D. Yu., Shkiro V. M., Ponomarev V. I. Equilibrium of products of self-propagating high-temperature synthesis. Doklady Physical Chemistry, 2004, vol. 394, no. 4-6, pp. 34-38. https://oi.org/10.1023/ B:DOPC.0000017998.96972.70

2. Nicolis G., Prigogine I. Self-organization in nonequilibrium systems: From dissipative structures to order through fluctuations. John Wiley \& Sons, 1977. 491 p.

3. Klubovich V. V., Kulak M. M., Rumyantseva I. N. Specific features of formation of bulk porous systems in the SHS process: Silicides. Vestsi Natsyyanal'nai akademii navuk Belarusi. Seryya fizika-technichnych navuk $=$ Proceedings of the National Academy of Sciences of Belarus. Physical-technical series, 2010, no. 4, pp. 5-10 (in Russian).

4. Klubovich V. V., Kulak M. M., Rumyantseva I. N. Specific features of formation of bulk porous systems in the SHS process: Carbides. Vestsi Natsyyanal'nai akademii navuk Belarusi. Seryya fizika-technichnych navuk = Proceedings of the National Academy of Sciences of Belarus. Physical-technical series, 2011, no. 1, pp. 5-10 (in Russian).

5. Klubovich V. V., Kulak M. M., Khina B. B. Ultrasound in SHS Processes. Minsk, Belarusian National Technical University, 2006. 279 p. (in Russian).

6. Image S.P. SYSPROG Software Development Company. Available at: https://sys-prog.com/en_US (accessed 16 August 2018).

7. Zeldovich Ya. B., Barenblatt G. I., Librovich V. B., Makhviladze G. M. The Mathematical Theory of Combustion and Explosions. New York, Consultants Bureau, 1985. 597 p.

8. Witusiewicz V. T., Bondar A. A., Hecht U., Rex S., Velikanova T. Ya. The Al-B-Nb-Ti system. I. Re-assessment of the constituent binary systems $\mathrm{B}-\mathrm{Nb}$ and $\mathrm{B}-\mathrm{Ti}$ on the basis of new experimental data. Journal of Alloys and Compounds, 2008, vol. 448, pp. 185-194. https://doi.org/10.1016/j.jallcom.2006.10.034 


\section{Информация об авторах}

Клубович Владимир Владимирович - академик Национальной академии наук Беларуси, доктор технических наук, профессор, заведующий лабораторией пластичности, Белорусский национальный технический университет (пр. Независимости, 65, 220013, Минск, Республика Беларусь); главный научный сотрудник, Физико-технический институт Национальной академии наук Беларуси (ул. Академика Купревича, 10, 220141, Минск, Республика Беларусь). E-mail: v_klubovich@tut.by

Кулак Михаил Михайлович - кандидат технических наук, старший научный сотрудник лаборатории физики металлов, Институт технической акустики Национальной академии наук Беларуси (пр. Генерала Людникова, 13, 210023, Витебск, Республика Беларусь). Е-mail: mmk_vit@tut.by

Хина Борис Борисович - доктор физико-математических наук, доцент, главный научный сотрудник, Физико-технический институт Национальной академии наук Беларуси (ул. Академика Купревича, 10, 220141, Минск, Республика Беларусь). E-mail: khina@tut.by

\section{Information about the authors}

Vladimir V. Klubovich - Academician of the National Academy of Sciences of Belarus, D. Sc. (Engineering), Professor, Head of the Laboratory of Plasticity, Belarusian National Technical University (65, Nezavisimosti Ave., 220013, Minsk, Republic of Belarus); Chief Researcher, Physical-Technical Institute of the National Academy of Sciences of Belarus (10, Academician Kuprevich Str., 220141, Minsk, Republic of Belarus). E-mail: v_klubovich@tut.by

Mikhail M. Kulak - Ph. D. (Engineering), Senior Researcher, Laboratory of Metal Physics, Institute of Technical Acoustics of the National Academy of Sciences of Belarus (13, General Ludnikov Ave., 210023, Vitebsk, Republic of Belarus). E-mail: mmk_vit@tut.by

Boris B. Khina - D. Sc. (Physics and Mathematics), Assistant Professor, Chief Researcher, Physical-Technical Institute of the National Academy of Sciences of Belarus (10, Academician Kuprevich Str., 220141, Minsk, Republic of Belarus).E-mail:khina@tut.by 